\title{
Influência da maltodextrina na curva glicêmica em praticantes de treinamento de força*
}

\section{Influence of maltodextrin on the curve glycemic in strength trainning practitioners}

\section{Wellington Fernando Silva ${ }^{1}$ Wallacy Rodrigues Alves ${ }^{2}$ Fábio Santana ${ }^{3}$ Márcio Rabelo Mota ${ }^{4}$ Darlan Lopes Farias ${ }^{5}$ Luis Inácio Silva ${ }^{6}$ Marco Santos Carvalho ${ }^{7}$}

Recebido em: 03/05/2015.

Aprovado em: 01/10/2015.

1 Pós graduando - Universidade Federal de Goiás - UFG; GRESPE - Grupo de Estudo e Pesquisa em Qualidade de Vida e Desempenho Humano;

2 Pós graduado - Centro Universitário de Volta Redonda - UNIFOA, Volta Redonda - RJ; GRESPE - Grupo de Estudo e Pesquisa em Qualidade de Vida e Desempenho Humano;

3 Professor Mestre - Universidade Estadual de Goiás - ESEFFEGO; UniEvangélica - Anápolis GO; GRESPE - Grupo de Estudo e Pesquisa em Qualidade de Vida e Desempenho Humano;

4 Professor Doutor - Centro Universitário de Brasília - UniCEUB - Brasília;

5 Professor Mestre - Centro Universitário de Brasília - UniCEUB - Brasília;

6 Graduado em Educação Física - Faculdade União de Goyazes - FUG, Trindade-GO; GRESPE - Grupo de Estudo e Pesquisa em Qualidade de Vida e Desempenho Humano;

Graduado em Educação Física - Faculdade União de Goyazes - FUG, Trindade-GO; GRESPE - Grupo de Estudo e Pesquisa em Qualidade de Vida e Desempenho Humano.

\section{Resumo}

O treinamento de força (TF) tornou-se parte integral no programa de condicionamento físico para atletas e praticantes de exercícios. O objetivo do estudo proposto neste artigo consistiu em identificar os efeitos da suplementação de maltodextrina referente à curva glicêmica em praticantes de TF. O estudo foi aprovado pelo comitê de ética e pesquisa da Faculdade União de Goyazes por meio do protocolo 011/2014-1. Vinte e um sujeitos do sexo masculino com, pelo menos, um ano de prática em TF participaram do estudo. Esses sujeitos foram divididos randomicamente a um dos dois grupos: grupo controle ( $\mathrm{G} 1, \mathrm{n}=11)$ e grupo experimental $(\mathrm{G} 2, \mathrm{n}=10)$. Desse modo, foram avaliados os níveis glicêmicos pré, durante e pós treino para analisar a curva glicêmica. Não houve redução significativa $(p>0,05)$ nos níveis glicêmicos da amostra, mas houve aumento quando comparados cada fase de coleta. Conforme o resultado obtido apresentado neste artigo, conclui-se que a maltodextrina, sendo utilizada como recurso ergogênico para TF, não apresentou valores significativos com relação ao grupo não suplementado.

Palavras-chave: Maltodextrina. Treinamento de Força. Glicose. Curva Glicêmica.

\begin{abstract}
The strength training (ST) has become an integral part of the fitness program for athletes and exercise enthusiasts. The aim of this study was to identify the effects of maltodextrin supplementation related to glycemic curve ST practitioners. The study was approved by the Research Ethics Committee Goyazes of Union College through the protocol 011 / 2014-1. Twenty-one male subjects with at least one year of practice in ST participated in the study. These subjects were randomly assigned to one of two groups: control group ( $\mathrm{G} 1, \mathrm{n}=11)$ and experimental group ( $\mathrm{G} 2, \mathrm{n}=10)$. Thus we evaluated the pre glucose levels, during and post workout to analyze the glycemic index. There was no significant reduction $(p>0,05)$ in blood glucose levels in the sample, but there was an increase compared each collection phase. As the result obtained in this study it is concluded that the maltodextrin is used as an ergogenic aid for ST no significant values with respect to the unsupplemented group.
\end{abstract}

Keywords: Maltodextrin. Strength Training. Glucose. Curve Glycemic. 


\section{Introdução}

O Treinamento de Força (TF) tornou-se parte integral no programa de condicionamento físico para atletas e praticantes de exercícios (SIMÃO, 2005). A popularidade desse método de treinamento é cada vez maior devido aos reconhecidos benefícios para a saúde e estética corporal.

Segundo o American College of Sports Medicine (ACSM, 2011), para obter benefícios com base na prática de exercícios físicos, é adequado que o indivíduo realize de duas a três sessões de treinamento por semana, com uma média 48 horas de intervalo entre as sessões de treinamento que são dependentes da intensidade e volume deste.

Simão (2004) identificou alguns benefícios relacionados a essa forma de treinamento tal como: redução dos níveis pressóricos de repouso, sobretudo em hipertensos, melhora no perfil de lipídeos sanguíneos, menor resposta da frequência cardíaca em repouso, melhora na tolerância à glicose, incremento de massa muscular e da densidade mineral óssea, atuação coadjuvante na redução da depressão e ansiedade.

Nesse contexto, tem se tornado comum o uso de estratégias nutricionais que variam em grau de eficiência, alimentos e componentes alimentares que podem melhorar a capacidade do indivíduo no exercício, e que têm sido descrito como suplementos alimentares ou auxílio ergogênico. Prestes et al. (2010) relata que a palavra "ergogênico" vem de origem grega, "érgon": trabalho e "gennan" que significa artifícios utilizados para melhora da performance.

Desse modo, a suplementação de carboidrato constitui uma boa forma de complementação alimentar para os praticantes de exercícios físicos que visam melhorar o desempenho e aumentar a massa muscular (FAYH et al., 2007), pois a ingesta desse nutriente contribui para a manutenção das concentrações de glicose plasmática, além de poupar o glicogênio muscular (PEREIRA et al., 2012).

Essa substância está diretamente relacionada ao índice glicêmico, que é classificado como marcador e indicador qualitativo da habilidade de carboidrato ingerido, em elevar os níveis glicêmicos na corrente sanguínea (SIU; WONG, 2004; MONDAZZI; ANCELLI, 2009).

$\mathrm{O}$ índice glicêmico constitui uma ferramenta utilizada na nutrição esportiva e sua manipulação pode otimizar o controle da hipoglicemia de rebote e a estimulação da oxidação lipídica, por mais disponibilidade de fontes de glicose durante o exercício e restauração do glicogênio muscular. Assim, demonstrando o impacto da ingestão de carboidrato na glicemia (PEREZ, 2012).

Assim, esse estudo teve como objetivo avaliar a curva glicêmica de um grupo suplementado com maltodextrina e um grupo controle para analisar qual influência da suplementação durante uma sessão de TF.

\section{Materiais e Métodos}

O estudo foi aprovado pelo Comitê de Ética e Pesquisa da Faculdade União de Goyazes por meio do protocolo 011/2014-1. Todos os participantes foram informados sobre os objetivos e os procedimentos da pesquisa, e os mesmos assinaram um Termo de Consentimento Livre e Esclarecido (TCLE), que apresenta todas as características da pesquisa, bem como os riscos e benefícios, além da preservação do anonimato dos participantes de acordo com as normas éticas da Resolução 466/12 do Conselho Nacional de Saúde (CNS, 2012).

A pesquisa proposta neste artigo foi realizada utilizando intervenção por meio de uma sessão de TF para análise da curva glicêmica no momento agudo, com característica quantitativa e experimental em praticantes de TF, sendo aplicado o "Método Cego", utilizando grupo experimental e grupo controle conforme gráfico - 1 (CERVO, 2007).

Como início dos procedimentos, foram verificadas a Frequência Cardíaca (FC) e a Pressão Arterial (PA), onde se determinou os valores pressóricos: sistólico e diastólico, além da FC, ambos mensurados em repouso, deste modo, com a associação entre FC e PA obtemos os valores do Duplo Produto (DP).

A determinação da Pressão Arterial Sistólica (PAS) e Diastólica (PAD) foi realizada pelo método oscilométrico, adotando-se a metodologia proposta pela VI Diretriz Brasileira de Hipertensão Arterial utilizando um medidor de pressão arterial Microlife ${ }^{\circledR}$ modelo BP3BT0-A automático, validado pela British Hipertension Society (CUCKSON et al., 2002). Com o indivíduo na posição sentada após 10 minutos de repouso, braço direito apoiado e ao nível do coração, braçadeiras apropriadas ao tamanho do braço, colocou-se a braçadeira do aparelho a cerca de $3 \mathrm{~cm}$ acima da fossa antecubital centralizando a bolsa de borracha sobre a artéria umeral. Os valores das 
medidas de PAS e PAD foram utilizados para o cálculo da Pressão Arterial Média (PAM) pela equação: $\mathrm{PAM}=\mathrm{PAD}$ $+[($ PAS - PAD $) \div 3]$, (TIBANA et al., 2011).

Para aferição da massa corporal e da estatura, utilizamos balança digital marca Welmy ${ }^{\circ}$ com estadiômetro acoplado com escala de graduação de 1 centímetro $(\mathrm{cm})$, sendo calibrada pelo Instituto Nacional de Metrologia, Qualidade e Tecnologia (INMETRO).

Para a coleta das circunferências, foram adotadas as orientações de McArdle et al (2011), em que utilizamos uma trena antropométrica metálica, marca Sanny ${ }^{\bullet}$ com escala de graduação a cada cm, em que esta foi aplicada levemente na superfície cutânea de maneira que fique justa, porém, não apertada, evitando a compressão da pele. De posse dessas variáveis, calculamos a Relação Cintura Quadril (RCQ) obtido por meio da divisão do perímetro da cintura pelo quadril e Índice de Adiposidade Corporal - IAC por meio da fórmula IAC $=[($ Circunferência da Cintura) / (estatura) $\left.{ }^{1.5}\right)$ - 18], que foi utilizada para caracterização da amostra (BERGMAN et al., 2011; TIBANA; BALSAMO; PRESTES, 2011).

A suplementação foi ofertada em período pós-prandial com 2 horas após o café da manhã, constituída por $0,5 \mathrm{~g} / \mathrm{kg}$ de maltodextrina por peso corporal para o G2, da marca Probiótica ${ }^{\circledR}$, diluída em $250 \mathrm{ml}$ de água. Para a pesagem dos carboidratos utilizamos uma balança de precisão da marca Camry modelo EK8022 (HERNANDEZ; NAHAS, 2009).

O TF foi realizado em única sessão utilizando 6 exercícios distribuídos da seguinte forma: Supino Reto, Leg Press, Puxada Pulley, Cadeira Extensora, Desenvolvimento e Mesa Flexora, realizando 03 séries de 10 repetições cada com carga a $80 \%$ da carga máxima estimada com intervalo de 60 segundos entre as séries, e execução vinte $\mathrm{x}$ vinte (20x20), ou seja, dois segundos na fase excêntrica e dois segundos na fase concêntrica, sem interrupção do movimento. Desse modo uma semana antes dos ensaios experimentais, uma carga de 10RM foi obtida para cada indivíduo, de acordo com os procedimentos de (SIMÃO et al., 2005).

A mensuração da glicemia capilar foi realizada em vários momentos: em jejum, 02 horas pós-prandial ao café da manhã, e na sessão de treino, sendo realizada na metade e ao final do treinamento, bem como durante uma hora de recuperação passiva após o treinamento, sendo coletados a cada 15 minutos para análise e determinação da curva glicêmica.
Para esse procedimento, foi adotado aparelho $A C$ $C U$ - $C H E K^{\circ}$ modelo Active e tiras reactivas da marca $R O-$ CHE para análise da glicemia. Para a coleta de sangue, foi realizada uma assepsia no lóbulo da orelha com álcool etílico hidratado 70\% INPM da marca Farmax $50 \mathrm{ml}$ e luvas cirúrgicas, além de lancetas picadoras inox estéril WILTEX ${ }^{*}$ para perfurar a orelha e Algodão Hidrófilo $25 \mathrm{~g}$ da marca Cremer. Destacamos que todo esse material utilizado foi descartável e de uso individual.

Figura 1 - Caracterização da pesquisa

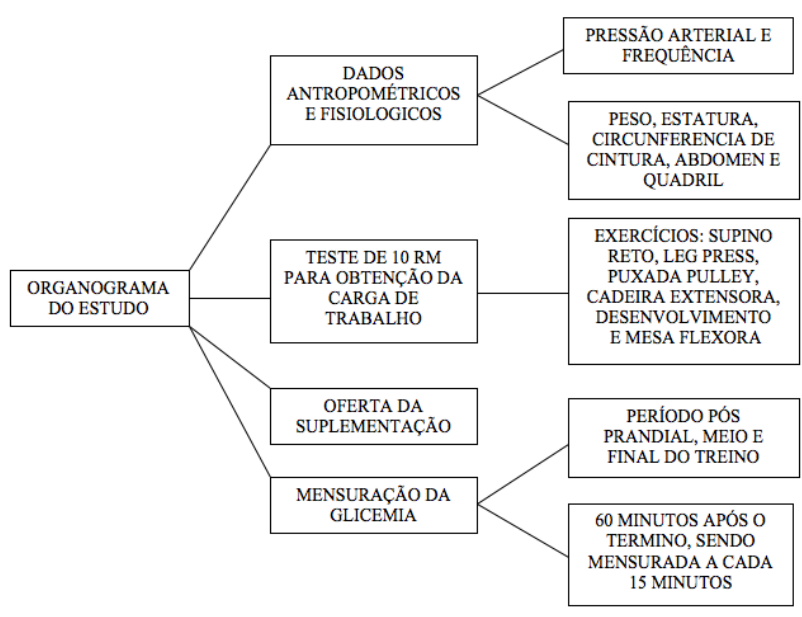

\subsection{População e amostra}

Para esse estudo foram utilizados como população praticantes de TF de uma academia de Goiânia. Dessa população foram selecionados um grupo de $(\mathrm{n}=$ 21) indivíduos para compor a amostra, sendo todos do sexo masculino com pelo menos um ano de prática de TF e com faixa etária entre 20 e 30 anos de idade e que buscam hipertrofia muscular como objetivo de treinamento. Estes foram divididos aleatoriamente em dois grupos: G1 ( $n=11)$ como grupo controle sem suplementação e G2 ( $\mathrm{n}=10)$ grupo experimental com utilização de Maltodextrina.

\subsection{Critérios de Inclusão e Exclusão}

Os critérios de inclusão foram: disponibilidade de horário para participação do estudo, ser do sexo masculino, não estar utilizando nenhum recurso ergogênico no mínimo a 3 meses, se enquadrar na faixa etária proposta, ter no mínimo de 1 ano de experiência em TF, não fazer uso de bebidas alcoólicas e tabaco, não ser portador de nenhuma doença ou lesões osteomioarticulares e apresentar atestado médico que está apto a praticar exercício físico, além de assinar o Termo de Consentimento Livre e Esclarecido para se tornar voluntário para a pesquisa. 
E como único critério de exclusão, não atender alguma das exigências acima citadas.

\subsection{Riscos e Benefícios}

A pesquisa apresenta baixo risco, visto que a amostra foi composta por indivíduos praticantes de TF, e o protocolo aplicado durante uma sessão de treinamento, está bem próximo do que eles executam rotineiramente. Porém, estes foram minimizados seguindo as orientações dos pesquisadores. Como benefício, foi oferecida ao grupo a oportunidade de utilização de suplemento na busca de potencializar o treinamento e melhorar seus respectivos rendimentos, além de contribuir com a comunidade acadêmica e científica.

\subsection{Controle de Parâmetros Dietético}

Os indivíduos participantes do estudo tanto do grupo controle G1 ( $\mathrm{n}=11)$ quanto do grupo experimental G2 $(n=10)$ foram orientados por uma nutricionista a não modificar seus hábitos alimentares nos dias que antecedem à coleta. Como a aplicação do protocolo foi realizada no período matutino, foi fornecido previamente aos voluntários, um cardápio de café da manhã composto por $60 \%$ de carboidrato $15 \%$ proteína e $25 \%$ de lipídeos, calculado por uma nutricionista, a fim de manter uma padronização de ingesta alimentar no controle dos grupos.

\subsection{Tratamento Estatístico}

Os dados foram analisados por meio de análise descritiva e teste " $t$ " de Student, para comparação das variáveis entre os grupos, além de uma ANOVA para medidas repetidas para comparar os dados pré e pós-sessão de treinamento, adotando como nível de significância ( $\mathrm{p}$ $\leq 0,05)$. Os dados foram estruturados e analisados utilizando o pacote estatístico software SPSS versão 20.0 for Windows.

\section{Resultados e discussão}

No que se refere às variáveis antropométricas, houve diferença significativa na RCQ entre os grupos mostrando a caracterização da amostra em questão. As demais variáveis não apresentaram valores significativos. Os resultados estão dispostos nas tabelas 1 e 2 .

Destaca-se que, apesar de ser um grupo homogêneo, houve diferença significativa no exercício de Leg Press, com valor de $(\mathrm{p}=0,0272)$ conforme Tabela 3 .
Ao analisar a influência do Treinamento de Força - TF na glicemia nos momentos durante o treinamento e ao final do treino, não apresentou valores significativos, isso pode ter ocorrido devido ao tempo de duração da sessão de treinamento que ficou em torno de (30,29 $\pm 2,22$ minutos) conforme figura 2 , e após o término do treinamento, ambos os grupos mostraram aumento da glicemia nas fases de 15',30' e 45' minutos. Isso pode ter ocorrido devido à necessidade de reposição de glicose na corrente sanguínea.

A tabela 1 caracteriza os valores da amostra pesquisada calem que apresentou diferença significativa de ( $\mathrm{p}<0,05)$ apenas na Relação Cintura Quadril (RCQ) entre G1 e G2. Desse modo podemos observar que os valores antropométricos que envolvem massa corporal, estatura e Índice de Adiposidade Corporal (IAC) não apresentaram diferença significativa $(\mathrm{p}>0,05)$.

Tabela-1 - Valores antropométricos para caracterização dos grupos avaliados.

\begin{tabular}{lccc}
\hline Variáveis & G1 - Controle & G2 - Experimental & Sig \\
\hline Massa Corporal - kg & $73,57 \pm 10,31$ & $76,81 \pm 8,26$ & 0,217 \\
\hline Estatura - m & $1,76 \pm 0,07$ & $1,77 \pm 0,07$ & 0,329 \\
IAC & $15 \pm 1,81$ & $15 \pm 2,43$ & 0,378 \\
\hline RCQ $-\mathrm{cm}$ & $0,77 \pm 0,07^{*}$ & $0,81 \pm 0,03^{*}$ & 0,049 \\
\hline
\end{tabular}

* Nível de Significância com ( $p \leq 0,05)$. IAC = Índice de Adiposidade Corporal. $\mathrm{RCQ}=$ Relação Cintura Quadril.

Pode-se analisar na Tabela 2 que as variáveis da Pressão Arterial Sistólica (PAS), Pressão Arterial Diastólica (PAD) e Pressão Arterial Média (PAM), não apresentaram diferença significativa estando dentro do padrão de normalidade segundo as VI Diretriz Brasileira de Hipertensão (2010), que destacam os valores limítrofes, similares aos valores apresentados em nossa pesquisa.

Tabela 2 - Respostas fisiológicas de repouso entre os grupos avaliados.

\begin{tabular}{|c|c|c|c|}
\hline Variáveis & G1 - Controle & G2 - Experimental & Sig \\
\hline $\mathrm{FC}-\mathrm{bpm}$ & $72,20 \pm 9,11$ & $71,18 \pm 9,81$ & 0,403 \\
\hline $\mathrm{PAS}-\mathrm{mmHg}$ & $123,4 \pm 12,00$ & $127,18 \pm 12,42$ & 0,243 \\
\hline PAD - mmHg & $76,4 \pm 9,69$ & $70,55 \pm 8,26$ & 0,075 \\
\hline PAM - mmHg & $92,07 \pm 9,68$ & $89,42 \pm 9,02$ & 0,262 \\
\hline Duplo Produto & $8899,5 \pm 1386,2$ & $9084,5 \pm 1725,5$ & 0,395 \\
\hline
\end{tabular}


Em relação ao Duplo Produto (DP), por ser uma variável dependente da relação da FC e PAS, que represente o consumo de oxigênio pelo miocárdico e denotando eficiência mecânica, também não apresentou significância entre os grupos G1 e G2, em que se destaca boa resposta das adaptações fisiológicas ao treinamento.

Ao analisar a tabela-3, que destaca os valores obtidos no teste de repetições máximas nos respectivos exercícios, observa-se que, apesar de ser um grupo homogêneo, houve diferença significativa entre G1 e G2 no exercício de Leg Press, com valor de $(p=0,0272)$.

Essa discrepância pode estar relacionada com a individualidade biológica da amostra, experiência dos indivíduos ( $\geq 1$ ano) na prática do Treinamento de Força, ou uma maior preferência por parte de alguns indivíduos na realização dos treinamentos no exercício Leg Press (SIMÃO et al., 2005).

Tabela 3 - Médias e desvio padrão do teste de Repetições Máximas.

\begin{tabular}{lccc}
\hline Variáveis & G1-Controle & G2 - Experimental & Sig \\
\hline Leg Press & $116,7 \pm 18,06^{*}$ & $137,8 \pm 27,60^{*}$ & 0,0272 \\
\hline Supino & $46,00 \pm 12,93$ & $48,55 \pm 9,04$ & 0,302 \\
\hline Extensora & $40,40 \pm 7,49$ & $40,91 \pm 5,84$ & 0,431 \\
\hline Puxada Pulley & $52,80 \pm 8,12$ & $52,91 \pm 5,58$ & 0,485 \\
\hline Flexora & $33,00 \pm 4,83$ & $25,09 \pm 7,08$ & 0,221 \\
\hline Desenvolvimento & $30,00 \pm 7,12$ & $26,55 \pm 5,80$ & 0,118 \\
\hline
\end{tabular}

Ao analisar a glicemia plasmática em 8 estágios, no figura-2 são apresentados os valores no qual foram subdividido da seguinte forma: jejum, durante treinamento, pós prandial, final do treino e dosagens nos 15, 30', 45’ e 60' minutos após o treinamento de força. Isto a fim de identificar a curva glicêmica do G1 - Grupo Controle e G2 - Grupo Experimental.

Figura 2 - Curva Glicêmica entre os G1 e G2 pré e pós exercício.

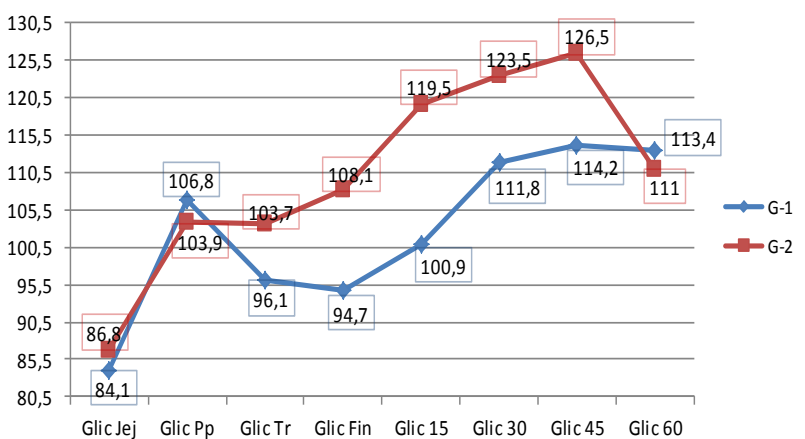

Observa-se que, na primeira e segunda dosagem, que se refere à glicemia em jejum G1 $(84,1 \pm 5,28 \mathrm{mg} /$ dl), G2 (86,8 $\pm 9,5 \mathrm{mg} / \mathrm{dl})$, e glicemia pós prandial G1 $(106,8 \pm 13,7 \mathrm{mg} / \mathrm{dl}), \mathrm{G} 2(103,9 \pm 19,6 \mathrm{mg} / \mathrm{dl})$, não obtivemos diferenças significativas entre os grupos, além de obedecer os padrões de normalidade referenciados (BACURAU, 2009).

Em um estudo realizado por Barbosa et al. (2010), os valores glicêmicos da amostra do estudo não apresentaram significância nesta fase da coleta, isso reforça que o controle nutricional foi bem aplicado nos momentos que antecederam as coletas nos grupos avaliados, seguindo um padrão sobre os parâmetros dietéticos da pesquisa.

Assim ao analisar a influência do TF na glicemia nos momentos durante o treinamento e ao final do treino, o G1 teve uma queda na glicemia em relação ao período pós prandial, com valores de $(96,1 \pm 11,6 \mathrm{mg} / \mathrm{dl})$ no meio do TF e $(94,7 \pm 23,2 \mathrm{mg} / \mathrm{dl})$ no final do TF.

O G2 apresentou uma queda na glicemia (103,7 $\pm 11,3 \mathrm{mg} / \mathrm{dl}$ ) em relação ao valor pós prandial, e, no final do TF, obteve valores de $(108,1 \pm 14,01 \mathrm{mg} / \mathrm{dl})$, não apresentando valores significativos com ( $p>0,05)$, isso pode ter ocorrido devido ao tempo de duração da sessão de treinamento que ficou em torno de $(30,29 \pm 2,22$ minutos), além da intensidade do treino, mesmo sendo controlado por meio do protocolo de 10RM. Em pesquisa realizada por Fayh et al. (2007), constataram que treinamentos com menos de 40 minutos é utilizado o glicogênio muscular como principal substrato energético, isso reforça nossos achados justificando a não significância desta fase da coleta.

Após o término do treinamento, ambos os grupos mostraram um aumento da glicemia nas fases de 15', 30' e $45^{\prime}$ minutos, isso pode ter ocorrido devido à necessidade de reposição de glicose na corrente sanguínea.

Sapata, Fayh e Oliveira. (2006) relatam que o aumento do glucagon estimula a glicogenólise hepática, causando aumento da glicemia plasmática, e isso contribui para o aumento apresentado em questão na fase pós treino, sendo um fator que pode ter influenciado a redução da glicemia na fase dos 60 minutos, em que a glicogenólise hepática já não era suficiente para suprir a necessidade energética, fazendo com que a glicemia plasmática retorna-se aos valores próximos dos iniciais. Contudo foi aplicada uma análise onde constatou-se que os valores finais $60^{\prime}$ pós sessão de TF apresentaram dife- 
rença significativa no G1 com valor $(p=0,0037)$ e no G2 valor de $(\mathrm{p}=0,0018)$.

\section{Conclusão}

Assim, este estudo teve como objetivo avaliar a curva glicêmica de um grupo suplementado com maltodextrina e um grupo controle para analisar qual influência da suplementação durante uma sessão de TF.

Desse modo conclui-se que a maltodextrina utilizada apenas uma vez como recurso ergogênico para o TF, não apresentou valores significativos ao grupo não suplementado, mostrando que a suplementação em relação à nossa amostra, não foi tão eficiente quanto aos níveis glicêmicos em um grupo com 1 ano de treinamento de força. Um possível fator para que os resultados não mostrassem valores significativos pode ter ocorrido devido ao tempo de duração da sessão de treinamento que girou em torno de (30,29 $\pm 2,22$ minutos).

No entanto, foram verificadas alterações na glicemia durante a pesquisa. Constatou-se que o controle nutricional pode ser uma ferramenta muito importante para a liberação de glicose na corrente sanguínea desde que seja bem controlado pelo nutricionista.

Desse modo, é interessante que novos estudos sejam realizados para entender melhor a suplementação de maltodextrina ao longo de uma sessão de TF, bem como outras variáveis como, influência hormonal, analisando as concentrações séricas de hormônios pancreáticos $\mathrm{e}$ adrenérgicos para elucidar os achados deste artigo, a fim de contribuir com a comunidade acadêmica e científica.

\section{Referências}

\section{AMERICAN COLLEGE OF SPORTS MEDICINE} (ACSM). Issues New Recommendations on Quantity and Quality of Exercise. Indianapolis, 2011. Available at: <http://www.acsm.org/about-acsm/media-room/ news-releases/2011/08/01/acsm-issues-new-recommendations-on-quantity-and-quality-of-exercise>. Access on: 5 may 2014.

BACURAU, R. F. Nutrição e suplementação esportiva. São Paulo: Phorte, 2009.
BARBOSA, L. C. et al. Diferença entre ingestão de carboidrato, placebo e curva glicêmica em teste progressivo em esteira (Conconi adaptado em esteira). Revista Brasileira de Nutrição Esportiva, São Paulo, v. 4, n. 20, p. 95-102, mar./abr., 2010.

BERGMAN, R. N. et al. A better index of body adiposity. Obesity, Malden, v. 19, n. 5, p. 1083-1089, maio 2011. doi: 10.1038/oby.2011.38.

CERVO, A. L. et al. Metodologia científica. 6. ed. São Paulo: Pearson Prentice Hall, 2007.

\section{CONSELHO NACIONAL DE SAÚDE. Resolução n. 196}

de 10 de outubro de 1996 versão 2012. Aprova as diretrizes e normas regulamentadoras de pesquisas envolvendo seres humanos. Plenário do Conselho Nacional de Saúde. Brasília, 2012. Disponível em: <http://conselho.saude. gov.br/web_comissoes/conep/aquivos/resolucoes/23_ out_versao_final_196_ENCEP2012.pdf >. Acesso em: 10 jun. 2014.

CUCKSON, A. C. et al. Validation of the Microlife BP 3BTO-A oscillometric blood pressure monitoring device according to a modified British Hypertension Society protocol. Blood Pressure Monitoring, Philadelphia, v. 7, n. 6, p. 319-324, dez. 2002. doi: 10.1097/00126097200212000-00005.

FAYH, A. P. T. et al. Efeitos da ingestão prévia de carboidrato de alto índice glicêmico sobre a resposta glicêmica e desempenho durante um treino de força. Revista Brasileira de Medicina do Esporte, Niterói, v. 13, n. 6, p. 416-420, nov./dez. 2007. doi: 10.1590/S151786922007000600012 .

HERNANDEZ, A.; NAHAS, R. M. Modificações dietéticas, reposição hídrica, suplementos alimentares e drogas: comprovação da ação ergogênica e potenciais riscos para a saúde. Revista Brasileira de Medicina e Esporte, Niterói, v. 15, n. 3, Supl. 0, p. 2-12, mar./abr. 2009. doi: $10.1590 /$ S1517-86922009000400001.

MCARDLE, W. D. et al. Fisiologia do exercício: energia, nutrição e desempenho humano. 7. ed. Rio de Janeiro: Guanabara-Koogan, 2011. 
MONDAZZI, L. M. D.; ANCELLI, E. M. D. Glycemic index in sport nutrition. Journal of the American College of Nutrition, New York, v. 28, Suppl. 4, p. 455-463, Aug. 2009. doi: 10.1080/07315724.2009.10718112.

PEREIRA, L. G. et al. Diferentes formas de suplementos de carboidrato durante o exercício: impactos metabólicos e no desempenho. Motricidade, Vila Real, v. 8, n. 2, p. 167-176, set. 2012.

PEREZ, R. Viva em dieta, viva melhor: aplicações práticas de nutrição. São Paulo: Phorte, 2012.

PRESTES, J. et al. Prescrição e periodização do treinamento de força em academias. São Paulo: Manole, 2010.

SAPATA, K. B.; FAYH, A. P. T.; OLIVEIRA, A. R. Efeitos do consumo prévio de carboidratos sobre a resposta glicêmica e desempenho. Revista Brasileira de Medicina do Esporte, Niterói, v. 12, n. 4, p. 189-194, jul./ago. 2006. doi: 10.1590/S1517-86922006000400005.

SIMÃO, R. et al. Influence of exercise order on the number of repetitions performed and perceived exertion during resistance exercises. The Journal of Strength and Conditioning Research, Champaign, v. 19, n. 1, p. 152156, fev. 2005.
SIMÃO, R. Treinamento de força na saúde e qualidade de vida. São Paulo: Phorte, 2004.

SIU, P. M.; WONG, S. H. Use of the glycemic index: effects on feeding patterns and exercise performance. Journal of Physiological Anthropology and Applied Human Science, Tokyo, v. 23, n. 1, p. 1-6, jan. 2004. doi: 10.2114/ jpa.23.1.

SOCIEDADE BRASILEIRA DE CARDIOLOGIA. VI Diretrizes Brasileiras de Hipertensão. Arquivos Brasileiros de Cardiologia, Rio de Janeiro, v. 95, n. 1, Supl. 1, p. 1-51, jul. 2010. doi: 10.1590/S0066-782X2010001700001.

TIBANA, R. A.; BALSAMO, S.; PRESTES, J. Associação entre força muscular relativa e pressão arterial de repouso em mulheres sedentárias. Revista Brasileira de Cardiologia, Rio de Janeiro, v. 24, n. 3, p. 163-168, maio/jun. 2011.

WILLIAMS, M. H. Nutrição: para a saúde, condicionamento físico, desempenho esportivo. 5. ed. São Paulo: Manole, 2005. 